Check for updates

Cite this: RSC Adv., 2018, 8, 9074

\section{A metabolomic strategy based on integrating headspace gas chromatography-mass spectrometry and liquid chromatography-mass spectrometry to differentiate the five cultivars of Chrysanthemum flower $\dagger$}

\begin{abstract}
Lin-Ning Zhang, ${ }^{a}$ Long Wang, ${ }^{a}$ Zi-Qi Shi, ${ }^{\text {tbc }}$ Ping Li (D) a and Hui-Jun Li (iD *a
The extreme complexity of the chemical composition of plant extracts requires an unbiased and comprehensive detection methodology to improve the potential of metabolomic study. The present work, taking five closely related cultivars of Chrysanthemum flowers as a typical case, attempts to develop a metabolomic strategy to find more markers of metabolites for precise differentiation based on headspace gas chromatography-mass spectrometry (HSGC-MS) and ultra-performance liquid chromatography coupled with quadrupole time-of-flight mass spectrometry (UHPLC-QTOF/MS). In detail, 53 batches of Chrysanthemum flower samples were collected and analyzed. The fusion of datasets from HSGC-MS and UHPLC-QTOF/MS was done in two different ways. After comparison, the fusion of the total peak area normalized metabolomic data was performed for multivariate statistical analysis. A total of 21 marker compounds (including 14 volatile and 7 nonvolatile metabolites) were identified, and a heatmap was employed for clarifying the distribution of the identified metabolites among the five cultivars. The results indicated that the integrated platform benefited the metabolomic study of medicinal and edible herbs by providing complementary information through fully monitoring functional constituents.
\end{abstract}

Received 20th December 2017 Accepted 18th February 2018

DOI: $10.1039 / c 7 r a 13503 c$

rsc.li/rsc-advances

\section{Introduction}

Chrysanthemum flower (CF), derived from the dried anthodium of Chrysanthemum morifolium Ramat. (Compositae family), is a traditional Chinese medicine commonly used for its pharmacological properties of dissipating cold, clearing heat, removing toxins from the body, and brightening the eyes. It has also been used as a healthcare tea for thousands of years in China. During the long history of cultivation, the species of cultivated $C$. morifolium have been categorized into five main cultivars, i.e. Hangbai Ju (HbJ), Gong Ju (GJ), Chu Ju (CJ), Bo Ju (BJ), and Huai Ju (HJ). Although these cultivars are officially documented in Chinese Pharmacopoeia (2015) under the singular item of "Juhua", ${ }^{1}$ they are individually labeled in herbal

${ }^{a}$ State Key Laboratory of Natural Medicines, China Pharmaceutical University, No. 24 Tongjia Lane, Nanjing, China. E-mail: cpuli@163.com; Fax: +86 258327 1379; Tel: +86258327 1379

${ }^{b}$ Affiliated Hospital of Integrated Traditional Chinese and Western Medicine, Nanjing University of Chinese Medicine, Nanjing, China

'Jiangsu Province Academy of Traditional Chinese Medicine, Key Laboratory of New Drug Delivery Systems of Chinese Materia Medica, Nanjing 210028, China. E-mail: shiziqi47@126.com; Fax: +8625 85608672; Tel: +862585608672

$\dagger$ Electronic supplementary information (ESI) available. See DOI: $10.1039 / \mathrm{c} 7 \mathrm{ra13503c}$ markets according to traditional customs. Due to their similar appearances, colors and aromas, intended adulteration and/or unintentional confusion can happen from time to time.

Pharmacological studies have revealed a wide spectrum of biological activity for $\mathrm{CF}$, such as antibacterial, antiinflammatory, anti-oxidant, anti-tumor, and phlegm-removing effects. Three kinds of chemical including volatile oils, caffeoylquinic acids and flavonoids have been reported in CF, the latter two types of which are considered as the biologically active components responsible for these activities. ${ }^{2,3}$ Therefore, out of the caffeoylquinic acids and flavonoids, chlorogenic acid, luteoloside and 3,5-O-dicaffeoylquinic acid are quantified as the quality control markers in Chinese Pharmacopoeia (2015). ${ }^{1}$ On the other hand, the composition of volatile oils is generally recognized as an important factor that represents the delicate natural aroma of CF, especially in floral tea. Due to the diverse germplasm resources of Chrysanthemum morifolium species, these CF cultivars highly differ in chemical composition, which consequently results in different medicinal functions as well as discriminatory application., ${ }^{4,5}$ During the past few decades, many chemical profiling methods have been developed for the purpose of quality evaluation of $\mathrm{CF}$, such as gas chromatography-mass spectrometry (GC-MS) for characterizing essential oils, ${ }^{6}$ and liquid chromatography-mass spectrometry 
(LC-MS) for characterizing flavonoids and/or caffeoylquinic acids. ${ }^{3,7}$ Additionally, chemical characterization coupled with chemometric analysis has also been employed to discriminate CF cultivars. ${ }^{\mathbf{8} 9}$ However, few study had carried out precise differentiation based on both volatile and non-volatile components.

In recent years, metabolomics has emerged as a valuable tool for the comprehensive profiling of metabolites in herbs. ${ }^{\mathbf{1 0 , 1 1}}$ Nuclear magnetic resonance (NMR) spectroscopy and MS are the main analytical techniques used in plant metabolomic studies. ${ }^{12}$ Although NMR spectroscopy can offer direct identification and quantification of abundant analytes, the NMR-based metabolomic approach suffers from a relatively low sensitivity compared with MS. ${ }^{13}$ In contrast, MS-based platforms, including GC-MS and LC-MS, have high sensitivity, high speed and broad application, giving rise to the most widely used metabolomic techniques. ${ }^{\mathbf{1 4 , 1 5}}$

GC and LC are complementary in the analysis of plant metabolites, which are naturally occurring with a broad polarity range, since the primary detection tool of choice for volatile molecules is GC while the preferred analytical technique for non-volatile molecules is LC. Considering the simultaneous occurrence of volatile oils, caffeoylquinic acids and flavonoids in CF, we attempted to integrate GC-MS and LC-MS in our study to find marker metabolites of $\mathrm{CF}$ for the purpose of precise differentiation, for a more comprehensive view. The proposed strategy is illustrated in Fig. S1 $\uparrow$ to deliver the methodology. Firstly, the chemical profiles of $\mathrm{CF}$, including volatile and nonvolatile metabolites, were globally characterized by a headspace GC-MS (HSGC-MS) method and an ultra-performance liquid chromatography coupled with quadrupole time-of-flight mass spectrometry (UHPLC-QTOF/MS) method, respectively. After the variables were extracted from the HSGC-MS and UHPLC-QTOF/MS raw data, fusion of these types of dataset was performed. Then, the combined datasets were subjected to multivariate data analyses including principle component analysis (PCA) and partial least squares-discriminant analysis (PLS-DA) for the discovery of marker metabolites with discriminant significance. The marker metabolites were screened by the variable importance in the projection (VIP) value and using the nonparametric Mann-Whitney $U$ test. Finally, the distribution of the marker metabolites in the five CF cultivars was displayed by a heatmap visualization.

\section{Materials and methods}

\subsection{Chemicals and reagents}

Ethyl decanoate (E101444-5, $\geq$ 99\%, Aladdin Chemistry Co. Ltd., China.) was used as the internal standard (IS-1) for HSGCMS analysis. Galangin (A0427, $\geq 98 \%$, Shanghai YaoYun Biotechnology Co. Ltd., China.) was used as the IS-2 for UHPLCQTOF/MS analysis. A mixture of $n$-alkanes (C8-C20, Lot: BCBK5370V) was purchased from Fluka (Buchs, Switzerland) to calculate the Kovats Indices (KI) of all the volatile constituents. Acetonitrile and formic acid of chromatographic grade were purchased from Anaqua Chemicals Supply Inc., Ltd. (Houston, TX, USA). Deionized water was prepared by a Milli-Q water purification system (Millipore, Bedford, MA, USA). All other reagents and chemicals were of analytical grade.

\subsection{Plant materials}

A total of 53 batches of five CF cultivars were purchased from herbal markets and local manufactures from Anhui, Zhejiang, Jiangsu and Henan provinces, China. The origins of the collected samples are recorded in Fig. S2. $\dagger$ The overall quality of all the samples, including macroscopic and microscopic characters, identified using thin layer chromatography (TLC) and HPLC assay, was in line with the criteria documented in Chinese Pharmacopoeia (2015). The voucher specimens were authenticated by the authors and deposited in the State Key Laboratory of Natural Medicines, Nanjing, China. All of the plant materials were finely pulverized and passed through a 60-mesh sieve before extraction.

\subsection{Gas chromatography-mass spectrometry experiments}

2.3.1 Sample preparation. Approximately $1.5 \mathrm{~g}$ of each CF sample was accurately weighed and directly sealed into a $20 \mathrm{~mL}$ HS vial. A quality control (QC) sample was prepared by pooling small aliquots of each sample to ensure a broad metabolite coverage ${ }^{15}$ An aliquot of $80 \mu \mathrm{L}$ of IS- 1 was added to the vial. The KI were calculated for all analytes using a homologous series of $n$-alkanes (C8-C20) on the HP-5MS column $(0.25 \mathrm{~mm} \times 30 \mathrm{~m}$, $0.25 \mu \mathrm{m}$, Agilent Technologies, Santa Clara, CA, USA).

2.3.2 Instrument parameters. HSGC-MS analysis was performed using an Agilent 7694E Headspace sampler (Agilent Technologies, Germany), connected to an Agilent 7890B series gas chromatograph (Agilent Technologies, Germany) coupled with an Agilent 5977A series mass spectrometer (Agilent Technologies, Germany) and equipped with a HP-5MS column.

The HS operating conditions were as follows: the equilibration time was $20 \mathrm{~min}$; the headspace oven, loop, and transfer line temperatures were 100,120 and $150{ }^{\circ} \mathrm{C}$, respectively; the shaking time was $2 \mathrm{~min}$ at low intensity; the injecting time was $2 \mathrm{~min}$.

GC operating conditions were as follows: the carrier gas (helium) was set at a flow rate of $1.0 \mathrm{~mL} \mathrm{~min}^{-1}$; the split ratio was $5: 1$; the column temperature program of GC was initially set at $50{ }^{\circ} \mathrm{C}$ for $1 \mathrm{~min}$, and was gradually increased to $100{ }^{\circ} \mathrm{C}$ at $3{ }^{\circ} \mathrm{C} \min ^{-1}$, then kept for $3 \mathrm{~min}$ before being gradually increased to $160^{\circ} \mathrm{C}$ at $10^{\circ} \mathrm{C} \mathrm{min}^{-1}$, and then being increased to $270{ }^{\circ} \mathrm{C}$ at $30^{\circ} \mathrm{C} \mathrm{min}^{-1}$; for MS detection, an electron ionization (EI) system was used with the ionization energy at $70 \mathrm{eV}$; the temperature of the ion source and the quadrupole temperature was $230{ }^{\circ} \mathrm{C}$ and $150{ }^{\circ} \mathrm{C}$, respectively; the mass range was $50-550$ amu in the full-scan acquisition mode with $3 \mathrm{~min}$ of solvent delay.

2.3.3 Data acquisition and processing. The HSGC-MS raw data including samples, QC samples and blanks were processed by MassHunter Qualitative Analysis B.06.00 for peak deconvolution. The parameters of the algorithm were set as follows: retention time $\left(t_{\mathrm{R}}\right)$ window size factor, 100; signal to noise ratio (SNR) threshold, 2; absolute height, 500; absolute area, 5000. The results were exported as ".cef" files and subsequently 
imported into the mass profiler professional software (version B02.00, Agilent Technologies, Santa Clara, CA, USA). A $t_{\mathrm{R}}$ window of $0.05 \mathrm{~min}$ and a match factor of $0.3 \mathrm{ppm}$ were set to align the peaks. The data were normalized using IS-1, and a transformation with a binary logarithm algorithm was conducted to reduce the differences among the values of each variable in the dataset. After filtering the variables using the algorithm of "filtering by frequency" and "one-way analysis of variance (ANOVA)", the resulting data were exported to Excel (Microsoft, Redmond, WA, USA) and then were subjected to unsupervised PCA and supervised PLS-DA using SIMCA-P 14.1 software (Umetrics $A B$, Umea, Sweden) to differentiate the samples and identify marker metabolites. At the same time, data normalized by the total peak area was also imported into SIMCA-P 14.1 software for multivariate statistical analysis.

\subsection{Ultraperformance liquid chromatography coupled with quadrupole time of flight mass spectrometry experiments}

2.4.1 Sample preparation. $50 \mathrm{mg}$ of each CF sample was accurately weighed into $4 \mathrm{~mL}$ of distilled water, and then ultrasonically extracted at $100 \mathrm{~Hz}$ for $30 \mathrm{~min}$. The extract was centrifuged at $5000 \mathrm{rpm}$ for $10 \mathrm{~min}$. The supernate $(200 \mu \mathrm{L})$ was mixed with methanol $(200 \mu \mathrm{L})$ and $80 \mu \mathrm{L}$ of $10 \mu \mathrm{g} \mathrm{mL}{ }^{-1}$ IS-2 solution in methanol by vortexing for $20 \mathrm{~s}$, and was then centrifuged at $13000 \mathrm{rpm}$ for $10 \mathrm{~min}$ at $4{ }^{\circ} \mathrm{C}$. Finally, the supernatant was used for UHPLC-QTOF/MS analysis. To evaluate the reproducibility of UHPLC-QTOF/MS during serial analysis, a QC sample was prepared by pooling small aliquots of each sample.

2.4.2 Instrument parameters. A Shimadzu LC30-AD HPLC system (Shimadzu, Kyoto, Japan) combined with a quadrupole time-of-flight mass spectrometer (Triple TOF 5600-1, AB SCIEX, Redwood City, CA, USA) was used. An Agilent Zorbax SB-C18 column (4.6 mm $\times 50 \mathrm{~mm}, 1.8 \mu \mathrm{m}$, Agilent Technologies, USA) with a column temperature maintained at $30{ }^{\circ} \mathrm{C}$ was employed. The mobile phase consisted of $0.1 \%$ formic acid (A) and acetonitrile (B), using a gradient elution of $10-20 \% \mathrm{~B}$ at $0-$ $2 \mathrm{~min}, 20-22 \% \mathrm{~B}$ at $2-9 \mathrm{~min}, 22-40 \% \mathrm{~B}$ at $9-16 \mathrm{~min}, 40-70 \% \mathrm{~B}$ at $16-17 \mathrm{~min}, 70-100 \% \mathrm{~B}$ at $17-18 \mathrm{~min}$, and $100 \% \mathrm{~B}$ at $18-$ $19 \mathrm{~min}$ with an equilibrium for $4 \mathrm{~min}$. The flow rate was set at $0.4 \mathrm{~mL} \min ^{-1}$ with an injection volume of $2 \mu \mathrm{L}$.

MS detection was performed using QTOF/MS in negative ionization mode with a DuoSpray ion source. The QTOF/MS was calibrated in high sensitivity mode and the automated calibration device system (CDS) was set to perform an external calibration every four samples using a calibration solution. The source parameters were optimized: collision voltage (CE), $50 \mathrm{eV}$; ion spray voltage floating (ISVF), $4500 \mathrm{~V}$; temperature, $500{ }^{\circ} \mathrm{C}$; nebulizing gas (GS1), 60 psi; heater gas (GS2), 60 psi; curtain gas, 35 psi. The MS was operated in full-scan TOF/MS (100-2000 $\mathrm{amu}$ ) and MS/MS mode (100-2000 amu) through dataindependent acquisition (DIA) in a single-run analysis. ${ }^{\mathbf{1 6}}$

2.4.3 Data acquisition and processing. Data acquisition was carried out using Analyst 1.6 software, and then imported to MarkerView version 1.1 (Applied Biosystems/MDS Sciex, Toronto, Canada) for preprocessing. A feature peak list was created directly from the raw data (.wiff) files with a subtraction offset of 10 scans, a minimum spectral peak width of $25 \mathrm{ppm}$, a minimum $t_{\mathrm{R}}$ peak width of 6 scans, and an SNR threshold of 100. Then, the UHPLC-MS data (.wiff) from multiple samples were imported into MarkerView using the following criteria: $t_{\mathrm{R}}$ tolerance, $0.5 \mathrm{~min}$; mass tolerance, $10 \mathrm{ppm}$; maximum number of peaks, 8000. Then, the isotope or monoisotope ions were excluded, normalization by IS- 2 and total peak area was further performed, and the peaks appearing in fewer than 2 samples with a response less than or equal to 50 were unused. The preprocessed data were exported to ".txt" files (Microsoft, Redmond, WA, USA), and were then subjected to unsupervised PCA and supervised PLS-DA using SIMCA-P 14.1 software.

\subsection{Multivariate statistical analysis}

Different normalization methods were conducted and compared, and the method with a finer resolution and prediction ability was chosen. The datasets of HSGC-MS and UHPLCQTOF/MS were normalized and then fused. Afterwards, the combined datasets were imported to SIMCA-P 14.1 software for multivariate statistical analysis. PLS-DA and PCA were applied to distinguish five CF cultivars and obtain potential markers (VIP > 1.5), and response permutation testing (RPT) was applied for assessing the goodness of fit.

Identification of the volatile components was performed by comparing the mass spectra with those recorded in the National Institute of Standards and Technology (NIST) mass-spectral library, and by comparing their KI with published literature.

Identification of the nonvolatile components was accomplished by using Formula Finder software and Chemspider online searching with built in PeakView 1.2, and later putatively confirmed by searching their accurate masses, empirical molecular formulas and MS/MS fragmentation behaviors against our in-house database and/or online metabolomic databases, including METLIN (http://www.metlin.scripps.edu) and Mass Bank (http://www.massbank.jp).

A nonparametric Mann-Whitney $U$ test was used to investigate the differences among the five cultivars in terms of these marker compounds $(p<0.05)$. Receiver operating characteristic (ROC) curves (SPSS 22.0) were utilized to analyze data with the purpose of evaluating the predictive power of the identified marker compounds. The discriminatory capability of each marker compound was ranked and visualized using a heatmap.

\section{Results and discussion}

\subsection{Optimization of extraction method and analysis conditions}

Steam distillation has been used for the collection of volatile compounds in terms of GC-MS for a long time. However, the tedious procedure is not suitable for high throughput experimentation. HS has proven to be a high-efficient sampling method for volatiles, with the advantages of reducing the complexity of sample pretreatment, shortening the manipulation time and showing general applicability to various samples in different states. ${ }^{17,18}$ Therefore, HS sampling coupled with the 
GC-MS method was utilized in this study. The HSGC-MS method was optimized by evaluating the effects of the relevant experimental parameters on the profiling of the volatile constituents. The equilibration time (10, 20 and $30 \mathrm{~min})$ and sampling amount $(1.0,1.5$ and $2.0 \mathrm{~g})$ were first investigated in the search for higher extraction efficiencies. It was found that HSGC-MS analysis with an equilibration time of $20 \mathrm{~min}$ and a sampling amount of $1.5 \mathrm{~g}$ showed a better result. Then, the temperatures of the headspace oven, loop, and transfer line were optimized as 100,120 and $150{ }^{\circ} \mathrm{C}$, respectively, with the ascending principle.

As for UHPLC-QTOF/MS analysis of organic acids and flavonoids in CF, the application of an RP-C18 column would inevitably neglect some hydrophilic compounds. Nonetheless, the present study attempted to detect as many caffeoylquinic acids and flavonoids as possible with optimization of the extraction method and chromatographic conditions. Firstly, ultrasonic extraction was chosen for being time-saving and for having good repeatability and the detailed parameters including extraction time and extraction solvent were optimized. Several reagents including methanol, water, 75\% methanol and $50 \%$ methanol were tried as the extraction solvent. Eventually, the procedure of water extraction-methanol precipitation was selected because of the dual functions of sample clean-up and enrichment of the target constituents. Since both caffeoylquinic acids and flavonoids possess carboxy groups and/or hydroxy groups, negative ion mode was generally selected for MS detection. However, before selection, we compared the LC/MS data generated from both positive-mode and negative-mode. Both TIC chromatograms showed similar peak shape and number, but the TIC chromatogram of negative ion mode showed a higher responsivity. For the purpose of getting useful fragment information for structural characterization, different CEs were tried (30, 40 and $50 \mathrm{eV}$ ). Finally, all the data were collected with a $\mathrm{CE}$ of $50 \mathrm{eV}$ to get fragment ion information.

\subsection{Repeatability and stability validation of the analyses}

To ensure the reliability of the analyses, the QC sample was analyzed before, during and after the sample analysis every day. The overlapping total ion current (TIC) chromatograms (Fig. S3 $\dagger$ ) of the QC samples demonstrated that acceptable variations occurred during the large-scale sample analyses. In the meantime, for each analytical method (HSGC-MS and
UHPLC-QTOF/MS), nine selected extracted ion chromatograms (EICs) in the QC samples were used to assess the system repeatability and stability. Based on the difference in polarity, several EICs from different eluting times were selected to be monitored. Relative standard deviations (RSDs) of the nine peaks from the HSGC-MS were $0.03-0.20 \%$ for the $t_{\mathrm{R}}$ and $0.97-$ $18.91 \%$ for the peak areas. RSDs of the nine peaks from the UHPLC-QTOF/MS were $0.02-0.76 \%$ for the $t_{\mathrm{R}}$ and $6.71-19.15 \%$ for the peak areas (Table S1 and S2 $\dagger$ ). At the same time, RSDs of the peak areas and the total peak area percentage (area sum\%) in the QC samples were used to verify the stability. Taking IS as an example, RSDs of IS-1 from the HSGC-MS were $16.31 \%$ for the peak areas and $7.04 \%$ for the area sum\% (Table S3 $\dagger$ ). RSDs of IS-2 from the UHPLC-QTOF/MS were $2.35 \%$ for the peak areas and $8.45 \%$ for the area sum\% (Table $\mathrm{S} 4 \dagger$ ). The $t_{\mathrm{R}}$, peak areas and area sum $\%$ of these selected peaks showed acceptable RSDs for both the HSGC-MS and UHPLC-QTOF/MS methods. Hence, these results supported their promising application to obtain high quality metabolomic data.

\subsection{Fusion of data and classification of five Chrysanthemum flower cultivars}

Representative HSGC-MS and UHPLC-QTOF/MS TIC chromatograms of different CF cultivars are illustrated in Fig. S4 and S5, $\dagger$ respectively. The TIC chromatograms showed the characteristic patterns (fingerprints) of each cultivar. After the serial data processing described in Sections 2.3.3 and 2.4.3, the normalized datasets (HSGC-MS and UHPLC-QTOF/MS) were fed to SIMCA-P 14.1 software independently for multivariate data analysis.

As an unbiased statistical approach, PCA was applied first. However, there were no obvious separation trends for the five groups of CF (Fig. S6 $\dagger$ ). The first three components of the models could only explain $32.0-55.6 \%$ of the variables. For this reason, PLS-DA, a supervised method, was applied for pattern recognition analysis. Fortunately, both the HSGC-MS-based model and the UHPLC-QTOF/MS-based model presented satisfying classification and prediction ability among the five CF cultivars (Table 1 and Fig. 1(a-d)). However, the two separation models did not consider the broad metabolite coverage in classification, which limited the ability to obtain a more complete overview of the metabolites in the samples. Due to the complementarity of GC and LC, it is worth considering merging the GC-MS and LC-MS measurements performed on the same samples to generate a comprehensive metabolomic profile.

Table 1 Parameters of the PLS-DA model based on HSGC-MS, UHPLC-QTOF/MS and the combined datasets from the two normalization methods, respectively

\begin{tabular}{llll}
\hline Normalization method & Type of dataset & $R^{2} X(\mathrm{cum})$ & $R^{2} Y(\mathrm{cum})$ \\
\hline Normalized by IS & HSGC-MS & 0.756 & 0.885 \\
& UHPLC-QTOF/MS & 0.550 & 0.914 \\
Normalized by total peak area & HSGC-MS & 0.814 & 0.831 \\
& UHPLC-QTOF/MS & 0.638 & 0.964 \\
Normalized by IS & Combined datasets & 0.535 & 0.824 \\
Normalized by total peak area & Combined datasets & 0.667 & 0.714 \\
\end{tabular}


With the purpose of evaluating the performance of the integration of the GC and LC methods, data fusion is the prerequisite step of data analysis. However, fusion of different MSbased metabolomic methods is not straightforward. The megavariate nature of the data (i.e., a very high variable to sample ratio) especially deserves attention. To eliminate this gap between GC-MS and LC-MS variables, fusion of the data can be done in different ways and on different levels. This also has repercussions for the fusion of metabolomics data. ${ }^{19}$

Herein, the HSGC-MS and UHPLC-QTOF/MS datasets were firstly normalized by respective IS and then fused to generate matrix 1. The integrated dataset was imported to SIMCA-P 14.1 for PLS-DA analysis. As a result, the model described $53.5 \%$ of the variation in $X\left(R^{2} X(\mathrm{cum})=53.5 \%\right)$ and $82.4 \%$ of the variation in the response $Y\left(R^{2} Y(\mathrm{cum})=82.4 \%\right)$, which also predicted $58.1 \%$ of the variation in the response $Y\left(Q^{2}\right.$ (cum) $=$ $58.1 \%$ ) (Table 1). Compared with all the models from before fusion, the classification and prediction ability was regrettably decreased. Thus, normalization with IS could not eliminate the large gap of variables between the HSGC-MS dataset and the UHPLC-QTOF/MS dataset. Finally, the peak areas of the HSGCMS and UHPLC-QTOF/MS chromatograms of the CF samples were normalized by the respective total peak area and summed up to generate matrix 2. Matrix 2 was also fed to SIMCA-P 14.1 for PLS-DA analysis. Consequently, this model provided finer resolution and prediction ability to distinguish these closely
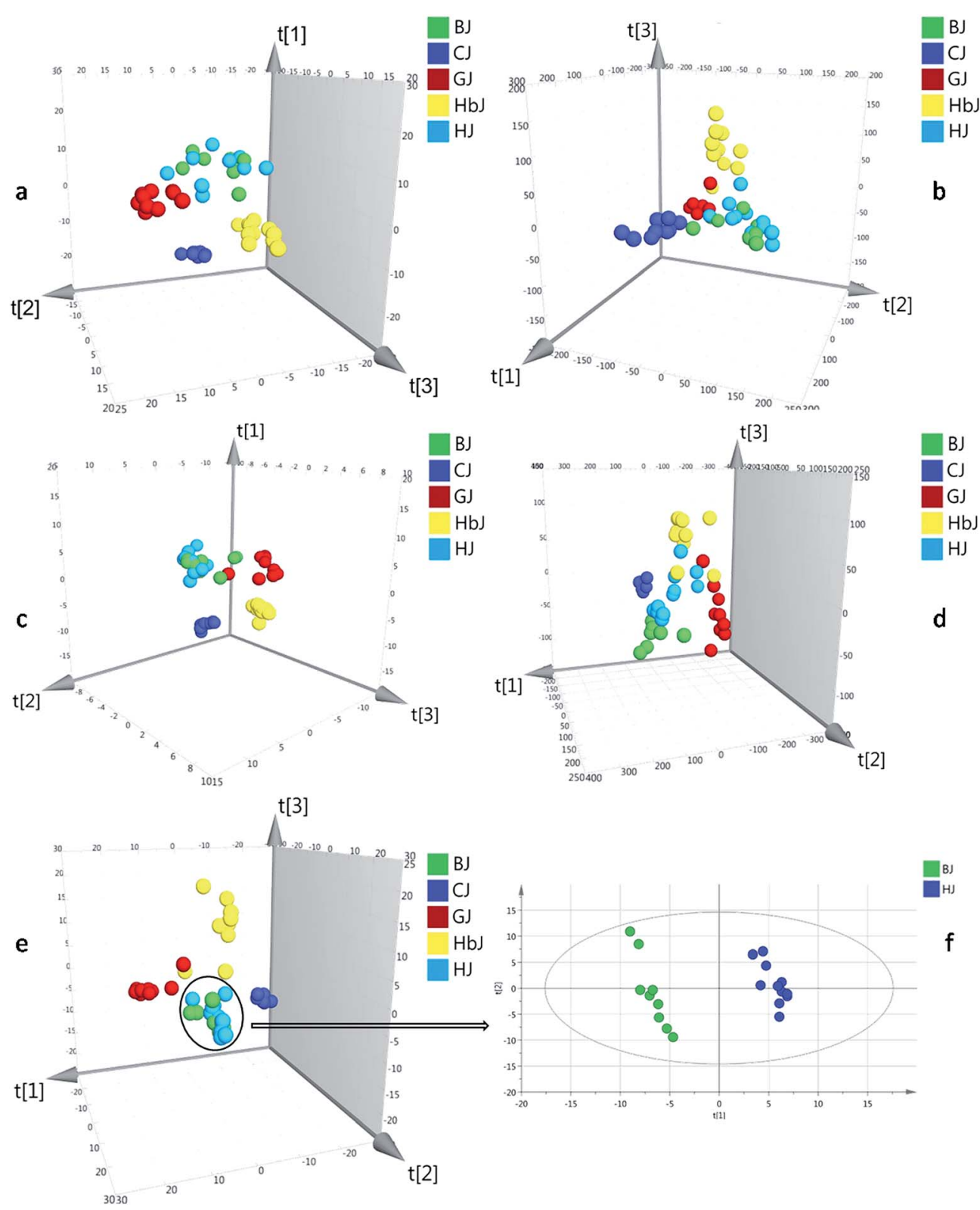

Fig. 1 The PLS-DA score plots for datasets of HSGC-MS and UHPLC-QTOF/MS. (a) HSGC-MS datasets normalized by an internal standard, (b) UHPLC-QTOF/MS datasets normalized by an internal standard, (c) HSGC-MS datasets normalized by the total peak area, (d) UHPLC-QTOF/MS datasets normalized by the total peak area, (e) combined datasets normalized by the total peak area, and (f) PLS-DA score plots of BJ and HJ samples exported independently for PLS-DA analysis. 
related CF groups with higher $R^{2} \mathrm{Y}$ and $Q^{2}\left(R^{2} \mathrm{Y}(\mathrm{cum})=0.967, Q^{2}\right.$ $($ cum $)=0.792$, Table 1). Based on the fused dataset, the score plot of PLS-DA is shown in Fig. 1e. From the 3D score plot, the five groups of $\mathrm{CF}$ samples were basically separated from each other, except for the BJ and HJ groups. We inferred that BJ and HJ had a closer phylogenetic relationship. This conjecture was supported by previous publications, in which the BJ cultivar was demonstrated to be originally introduced from $\mathrm{HJ}^{.20,21}$ Once the variables of the $\mathrm{BJ}$ and $\mathrm{HJ}$ samples were extracted from matrix 2, and the extracted variables were treated as a new matrix and exported independently for PLS-DA analysis, obvious separation was observed as shown in Fig. 1f. This finding indicated that interregional variation in metabolites could also be precisely distinguished.

RPT was applied for assessing the goodness of fit (Fig. S7 $†$ ). As a result, the validity of the combined model was proven. In the permutation test, all of the $R^{2}$ (cum) and $Q^{2}$ (cum) values calculated from the permuted data were lower than the original ones in the validation plot. The $Q^{2}$ (cum) intercepted the $y$-axis at 0.290 .

\subsection{Marker metabolites discovery and identification}

The variables that contributed to the observed separation were selected based on the parameter VIP value. The VIP-value threshold cut off of the metabolites was set to 1.5, and any variables above this threshold were filtered out as potential target biomarkers. Next, a nonparametric Mann-Whitney U test was carried out to compare the differences of potential markers in different cultivars of CF. Variables without significant differences among $\mathrm{BJ}, \mathrm{CJ}, \mathrm{GJ}, \mathrm{HbJ}$ and $\mathrm{HJ}(p>0.05)$ were excluded, while the variables with a VIP-value $>1.5$ and $p<0.05$ were screened as marker compounds. In total, 28 variables were screened as marker compounds. Among them, 16 variables were from the volatile components and 12 variables were from the nonvolatile components.
The identification of the volatiles was mainly based on the MS comparison with the standards in the NIST library, the KI obtained in this study, and the reported values in the literature with the same or equivalent columns. The reproducibility of the fragment patterns of the HSGC-MS experimental data is credible and fits well with the NIST database. ${ }^{22-28}$ Therefore, most of the 15 HSGC-MS variables were putatively identified except one. The identified metabolites are summarized in Table 2 with their corresponding $t_{\mathrm{R}}$ values, VIP values, $\mathrm{m} / \mathrm{z}$ values of the ions, matching degree with NIST, experimental KI and reported KI values, identified names, chemical formulas and CAS numbers.

Unlike GC-MS, a sophisticated LC-MS database for plant metabolites has not yet been constructed because of the extensive compound testing and limited reproducibility. ${ }^{29}$ Therefore, the identification of the screened LC-MS variables was largely based on previous phytochemical studies. In this study, peakview software was used to identify metabolites through searching the accurate masses, empirical molecular formulas and MS fragmentation behaviors against our in-house database and against online metabolomic databases, including the PubChem compound database (http://www.ncbi.nlm.nih.gov), METLIN (http://www.metlin.scripps.edu/) and MassBank (http:// www.massbank.jp/). Here, the processes of identification are briefly illustrated below by taking the ion of $\mathrm{m} / \mathrm{z} 338.0776$ as an example. As shown in Fig. 2, 2b shows two signals at $m / z 677.1726$ and $m / z 338.0823$, which correspond to the mono- and doublecharged molecular ions of a species with the molecular weight of $678.5930\left(\mathrm{C}_{34} \mathrm{H}_{30} \mathrm{O}_{15}\right)$. Fig. 2c shows a series of fragmentation ions, closely related with the ion at $\mathrm{m} / \mathrm{z} 677.1726$, at $\mathrm{m} / \mathrm{z} 515.1172$, $353.0902,191.0563,179.0350,173.0458,161.0258$ and 135.0466. Of these, the product ions at $\mathrm{m} / \mathrm{z} 191.0563$ (loss of caffeic moiety) and $\mathrm{m} / \mathrm{z} 173.0458$ (dehydrated quinic moiety) correspond to the quinic moiety. The ions at $\mathrm{m} / \mathrm{z} 179.0350$ (loss of quinic moiety) and $\mathrm{m} / \mathrm{z}$ 161.0258 (dehydrated caffeic moiety) were indicative of the caffeic moiety. Therefore, $m / z 677.1726$ was identified as the protonated

Table 2 NIST library-based putative identification of the volatile metabolites analyzed by HSGC-MS ${ }^{a, b}$

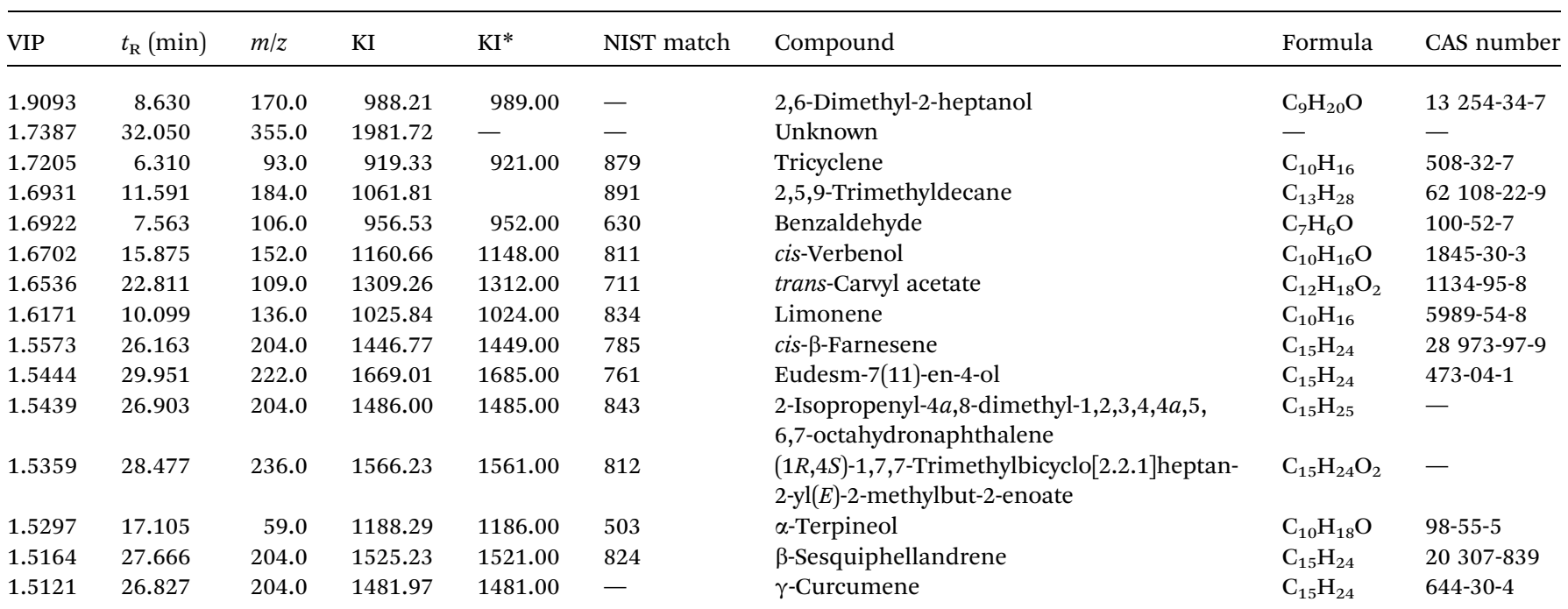

${ }^{a} \mathrm{KI}$ : experimental retention index. ${ }^{b} \mathrm{KI}^{*}$ : retention index from the literature. 

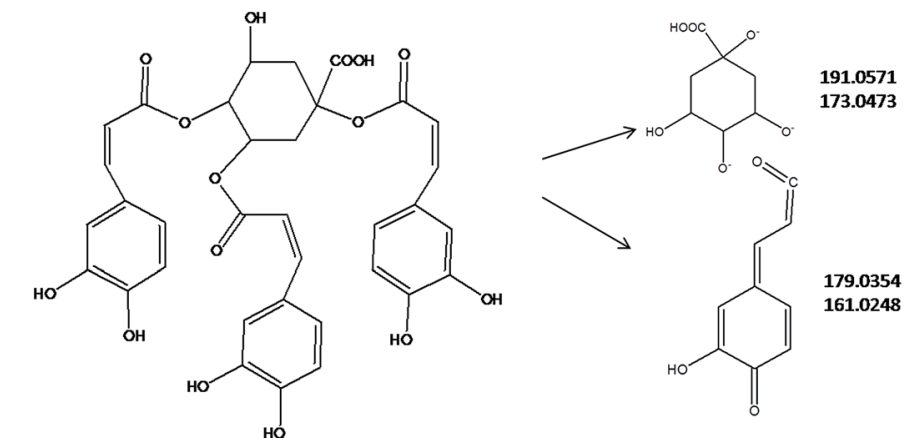

a
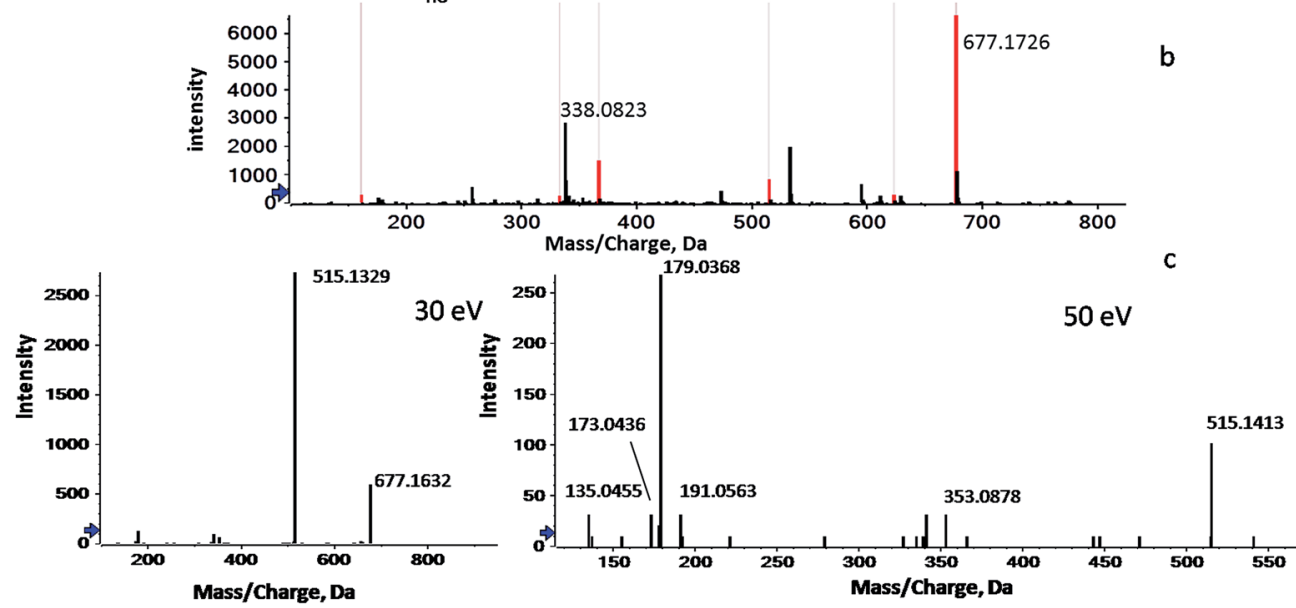

Fig. 2 The flow diagram of the identification of a marker compound (taking the ion of $\mathrm{m} / \mathrm{z} 338.0776$ as an example). (a) The structure of 1,3,4-triCQA, (b) mono- $(\mathrm{m} / \mathrm{z}=677.1726)$ and double-charged $(\mathrm{m} / \mathrm{z}=338.0823)$ molecular ions in a precursor spectrum, and (c) a series of fragmentation ions closely related with the ion at $\mathrm{m} / \mathrm{z} 677.1726$ (30 and $50 \mathrm{eV}$ ).

tri-caffeoylquinic acid (tri-CQA) because of the ions at $m / z 515.1172$ and $m / z 353.0902$ (loss of caffeic moiety). According to the published literature, $m / z 173.0458$ present in the product ion spectrum is indicative of acylation at position C4 of CQA. Employing the information above, $\mathrm{m} / \mathrm{z} 338.0823$ could be attributed to 1,3,4-triCQA (Fig. 2a). ${ }^{30-32}$ A similar workflow was applied to identify the 12 discriminant ions selected in the experiments. In this way, 7 discriminant ions were tentatively identified. The other 5 ions still remain unknown due to a lack of relevant reference standards and literature even though huge efforts have been made. The details of the 12 potential markers, including $t_{\mathrm{R}}$, types of ion, ppm errors, characteristic fragment ions, identified names and formulas, are summarized in Table 3.

ROC curve analysis was used for assessing the specificity of the marker compounds in the distinction of different CFs. As shown in Fig. S8 and Tables S5-8, $\dagger$ the area under the curve (AUC) for CJ-HbJ, CJ-GJ, and GJ-HbJ ranged from 0.9 to 1.0, while the AUC for BJ-HJ was 0.5 to 0.7 , the results of which are also consistent with the PLS-DA analysis. The results indicated that the identified biomarkers had a powerful diagnostic performance for most of the CFs.

\subsection{Distribution of marker compounds in five Chrysanthemum flower cultivars}

Once the marker compounds had been screened and putatively identified, the next step was to clarify the distribution of the marker compounds in different CF cultivars. This information is always of significance for phytochemotaxonomy. Therefore, a heatmap visualization was performed to obtain a deeper understanding of the distribution of the marker compounds. The relative concentration trends of the marker compounds in all the test samples are illustrated in Fig. 3. Although the genetic relationship among these five cultivars seemed to be very complicated due to ex situ introduction and mutual grafting during the long cultivation history, ${ }^{21}$ the heatmap clearly indicates that there are high chemical heterogeneities. Very clearly, among the identified marker compounds, the flavonoids including isoquercetin-2-O-(6'-acetyl-glucoside)-2'-glucoside, apigenin-7-caffeoylglucoside, dihydroluteolin-7-O-raffinoside, and the volatile oils such as $\gamma$-curcumene, $(1 R, 4 S)$-1,7,7-trimethylbicyclo[2.2.1] heptan-2-yl $(E)$-2-methylbut-2-enoate, 2-isopropenyl-4a,8-dimethyl-1,2,3,4,4a,5,6,7-octahydronaphthalene, eudesm-7(11)-en-4-ol, cis- $\beta$-farnesene and $\beta$-sesquiphellandrene were mainly distributed in CJ, while these markers could be rarely found in other cultivars. Similarly, BJ was chemically characterized by the presence of the following four volatile constituents: $\alpha$-terpineol, cis-verpineol, trans-carvyl acetate and benzaldehyde. HbJ was characterised by 2,6-dimethyl-2heptanol and limonene, HJ by two caffeoylquinic acids $(1,3,4-$ tri-caffeoylquinic acid and 5-acetyl-1,3,4-tri-caffeoylquinic acid) and two volatiles (limonene and $\alpha$-terpineol), and GJ by two volatiles viz. tricyclene and benzaldehyde. 
Table 3 Putatively identified nonvolatile metabolites detected by UHPLC-QTOF/MS

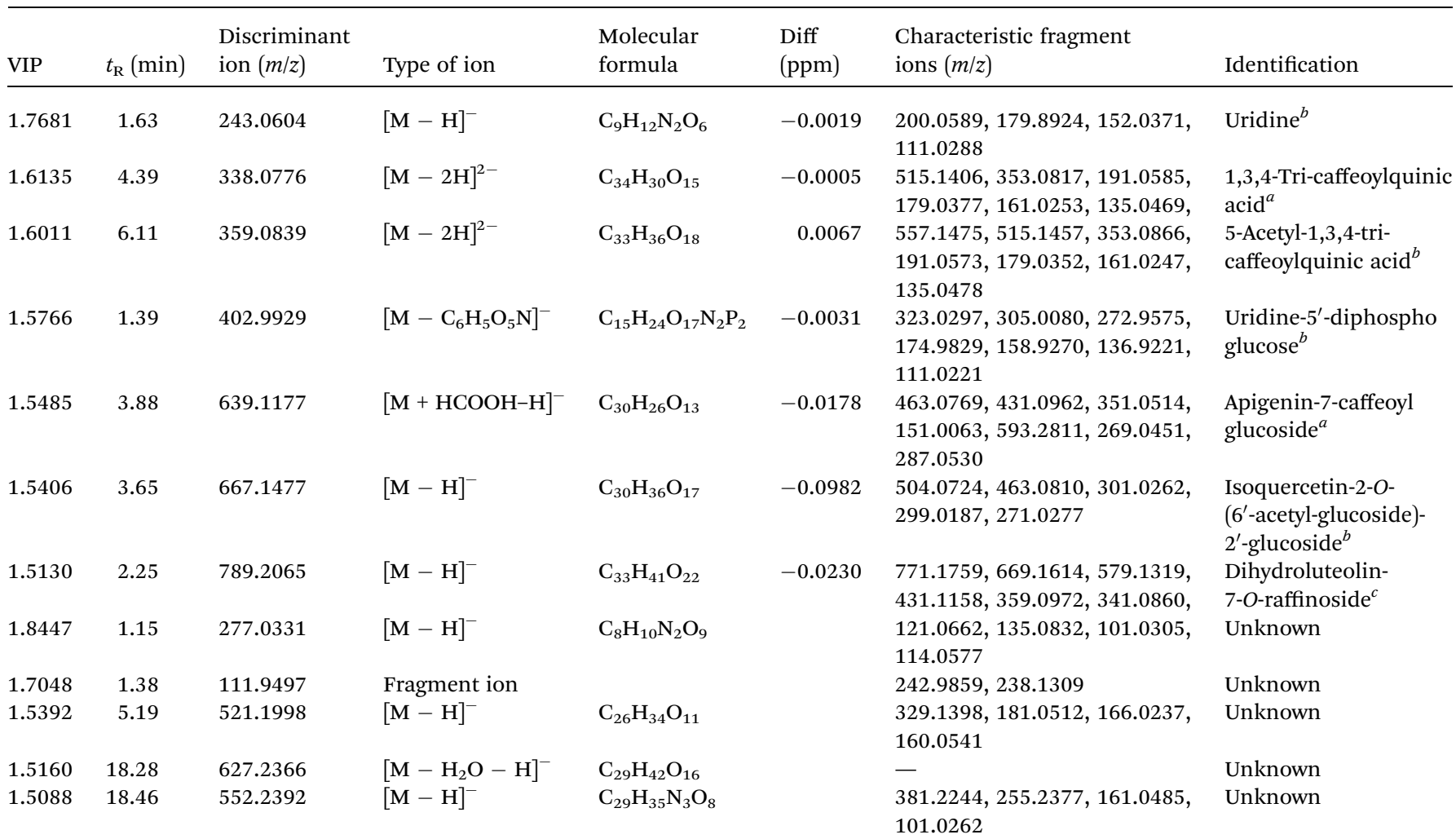

${ }^{a}$ Reported in the plant previously. ${ }^{b}$ Identified tentatively from the UHPLC-QTOF/MS data and the online metabolomics databases. ${ }^{c}$ Identified tentatively from the UHPLC-QTOF/MS data.

As one of the most famous geo-herbs indigenous to the Wuzhi and Wenxian counties of the Henan province, $\mathrm{HJ}$ has been considered to be the father of medicinal CF cultivars. ${ }^{33} \mathrm{BJ}$ was also a commonly medicinally-used cultivar, and its characteristic distribution of the marker compounds was more similar to that of $\mathrm{HJ}$ than to the other CF cultivars. The cultivars of GJ and $\mathrm{HbJ}$ are often consumed as health tea, and according to the clinical practice of traditional Chinese medicine (TCM), the former is good at clearing liver heat while the latter is good at clearing lung heat. ${ }^{34}$ Although the specific compounds responsible for the different efficacies remain unclear, a great discrepancy between GJ and HbJ could be seen in the heatmap.

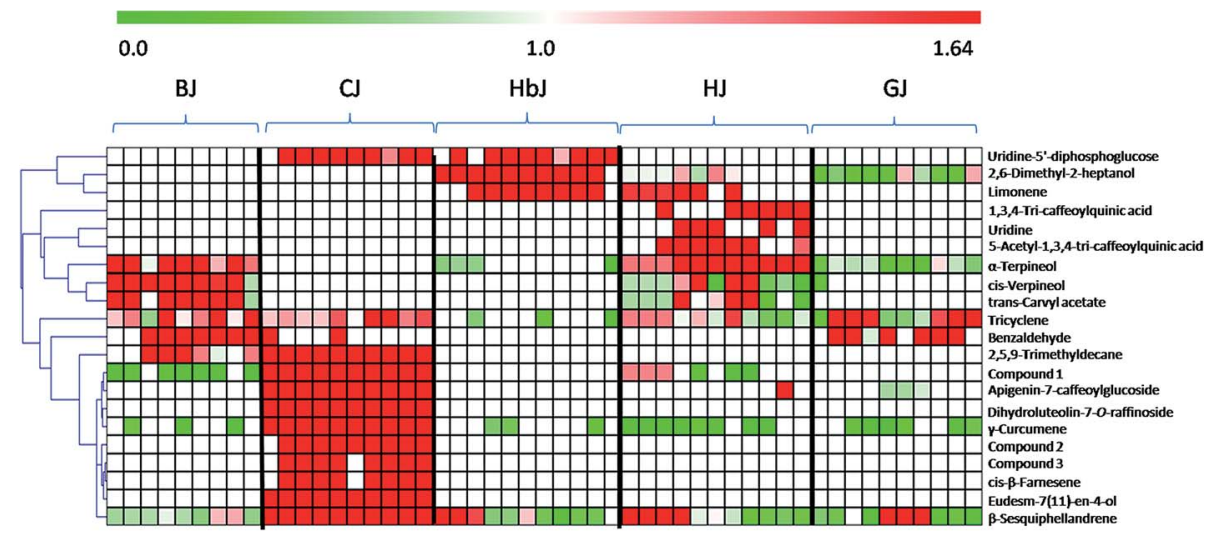

Fig. 3 The heatmap of the identified marker compounds: the relative concentration trends of the potential chemical markers in all the test samples using heatmaps are illustrated, in which the shade of the color indicates the different concentration levels of a chemical. The more red or more green the color is, the higher or lower the relative concentration level is, respectively. Compound 1: isoquercetin-2-O-(6'-acetylglucoside)-2'-glucoside. Compound 2: (1R,4S)-1,7,7-trimethylbicyclo[2.2.1]heptan-2-yl(E)-2-methylbut-2-enoate. Compound 3: 2-isopropenyl4a,8-dimethyl-1,2,3,4,4a,5,6,7-octahydronaphthalene. 
As for CJ, it was clearly distinguishable from the other cultivars, suggesting a distant genetic relationship with the other CF cultivars. Meanwhile, the quality consistency among the different batches for this cultivar seemed to be considerably higher than for the others, which might be due to its relatively fixed geo-origin. This consistent quality of CJ guarantees its irreplaceable medicinal value, which is supported by previous publications. $^{33}$

These experimental data, taken together, indicate that this metabolomic platform based on the fusion of HSGC-MS and UHPLC-MS datasets is a powerful way to visualize the difference in closely related species of medicinal herbs.

\section{Conclusions}

In this study, a metabolomic strategy based on the fusion of HSGC-MS and UHPLC-MS datasets was firstly proposed to differentiate the five CF cultivars. HSGC-MS was applied for the analysis of volatile metabolites, and UHPLC-MS provided a suitable complementary method to effectively characterize nonvolatile substances. A total of 21 marker compounds (14 volatile and 7 nonvolatile) were putatively identified. A heatmap was employed for clarifying the distribution of identified metabolites, and this could be useful for phytochemotaxonomy studies of CF species. More significantly, this integrated metabolomic strategy might be expanded to resolve other more complicated issues in plant systems where a full scale of metabolites instead of specifically targeted metabolites are really involved.

\section{Conflicts of interest}

The authors have declared no conflict of interest.

\section{Acknowledgements}

This work was supported by the National Natural Science Foundation of China (No. 81322051, 81130068 and 81603500). We also thank the Project Funded by the Priority Academic Program Development (PAPD) of Jiangsu Higher Education Institutions.

\section{References}

1 Chinese Pharmacopoeia Commission, Pharmacopoeia of the People's Republic of China 2015, Chinese Medical Science and Technology Press, Beijing, 2015, vol. 1, pp. 310-311.

2 X. Y. Hang, L. H Duan and D. Zhao, Lishizhen Med. Mater. Med. Res., 2008, 7, 1702-1704.

3 Y. R. Niu, Y. R. Yin, L. L. Yin, S. H. Luo, J. Dong, H. Wang, Y. K. Hashi and S. Z. Chen, Phytochem. Anal., 2013, 24, 59-68.

4 D. L. Li, J. Anhui Agric. Sci., 2010, 7, 3444-3446.

5 X. R. Liang, H. Wu and W. K. Su, Food Anal. Method, 2014, 7, 197-204.

6 H. N. Sun, J. Zhang, Q. Q. Fan, X. Y. Qi, F. Zhang, W. M. Fang, J. F. Jiang, F. D. Chen and S. M. Chen, Molecules, 2015, 20, 5346-5359.

7 Y. J. Chen, C. Y. Lin, S. S. Cheng and S. T. Chang, J. Agric. Food Chem., 2015, 63, 810-820.
8 Y. Y. Xie, J. L. Qu, Q. L. Wang, Y. Wang, M. Yoshikawa and D. Yuan, J. Agric. Food Chem., 2012, 60, 12574-12583.

9 Y. Q. Han, L. Q. Wang, X. H. Ying, J. M. Peng, M. Jiang, G. Bai and G. A. Luo, J. Ethnopharmacol., 2015, 174, 387-395.

10 L. X. Duan, T. L. Chen, M. Li, M. Chen, Y. Q. Zhou, G. H. Cui, A. H. Zhao, W. Jia, L. Q. Huang and X. Q. Qi, Mol. Plant, 2012, 2, 376-386.

11 L. X. Guo, R. Li, K. Liu, J. Yang, H. J. Li, S. L. Li, J. Q. Liu, L. F. Liu and G. Z. Xin, J. Chromatogr. A, 2015, 1425, 129-140.

12 L. Wang, L. F. Liu, J. Y. Wang, Z. Q. Shi, W. Q. Chang, M. L. Chen, Y. H. Yin, Y. Jiang, H. J. Li, P. Li, Z. P. Yao and G. Z. Xin, Anal. Chim. Acta, 2017, 997, 28-35.

13 G. A. N. Gowda and D. Raftery, J. Magn. Reson., 2015, 260, 144-160.

14 D. H. Robert, New Phytol., 2006, 169, 453-468.

15 D. K. Lee, M. H. Yoon, Y. P. Kang, J. Yu, J. H. Park, J. Lee and S. W. Kwon, Food Chem., 2013, 141, 3931-3937.

16 Z. Y. He, Y. P. Xu, L. Wang, Y. Peng, M. Luo, H. Y. Cheng and X. W. Liu, Food Chem., 2016, 196, 1248-1255.

17 M. C. G. Mas, L. M. G. Rian, C. Alfaro, J. L. Rambla and A. I. Padilla, Int. J. Food Sci. Technol., 2009, 44, 510-518.

18 L. M. Chen, A. Kotani, F. Kusu, Z. M. Wang, J. J. Zhu and H. Hakamata, Chem. Pharm. Bull., 2015, 63, 25-32.

19 A. K. Smilde, M. J. Werf, S. Bijlsma, B. J. C. Werff and R. H. Jellema, Anal. Chem., 2005, 77, 6729-6736.

20 L. Liu, Q. S. Guo and W. B. Xu, China J. Chin. Mater. Med., 2008, 33, 2891-2895.

21 D. Q. Wang, S. J. Liu and Y. M. Liang, J. Anhui TCM Coll., 2001, 20, 45-48.

22 R. P. Adams, Identification of essential oil components by gas chromatography/quadrupole mass spectroscopy, Allured Publishing Corporation, Appendix I, Carol Stream, IL, 2001.

23 X. D. Ma, W. W. Mao, P. Zhou, P. Li and H. J. Li, Microsc. Res. Tech., 2015, 78, 633-641.

24 D. S. B. Alexandre and S. M. D. Morais, Ind. Crops Prod., 2015, 76, 557-564.

25 G. Flamini, P. L. Cioni and I. Morelli, J. Agric. Food Chem., 2013, 51, 2267-2271.

26 L. Sheng, L. Y. Wen, C. X. Zhao and Y. Z. Liang, J. Instrum. Anal., 2008, 27, 84-87.

27 P. A. Robert, J. Am. Soc. Mass Spectrom., 2005, 16, 1902-1903.

28 R. C. H. De Vos, S. Moco, A. Lommen, J. J. B. Keurentjes, R. J. Bino and R. D. Hall, Nat. Protoc., 2007, 2, 778-791.

29 S. Moco, R. J. Bino, O. Vorst, H. A. Verhoeven, J. Groot, T. A. Beek, J. Vervoort and C. H. R. De Vos, Plant Physiol., 2006, 141, 1205-1218.

30 M. N. Clifford, S. Knight and N. Kuhnert, J. Agric. Food Chem., 2005, 53, 3821-3832.

31 M. N. Clifford, S. Knight and N. Kuhnert, J. Agric. Food Chem., 2003, 51, 2900-2911.

32 S. T. Wang, H. Yang, W. Gao, H. J. Li and P. Li, J. Pharm. Biomed. Anal., 2016, 119, 91-98.

$33 \mathrm{~J}$. Zhou, Studies on the problem of origin of Chinese garden Chrysanthemum, Beijing Forestry University, Beijing, 2009.

34 L. Zhuo, X. Li and G. J. Qiu, J. Neijiang Norm. Univ., 2008, 23, 104-105. 